Trab. Ling. Aplic., Campinas, 44(2): 215-231, Jul./Dez. 2005

\title{
INFLUENCES INTERNATIONALES SUR LA LEXICOGRAPHIE PEDAGOGIQUE DU FLE
}

\author{
BINON J., VERLINDE S., SELVA T., (GRELEP) \\ Institut interfacultaire des langues vivantes \\ Université Catholique de Leuven (Belgique)
}

"Pour l'apprenant d'une langue étrangère rien ne va de soi, rien n'est prévisible". Schneider $(1998,73)$

\begin{abstract}
RESUME
L'article commence par dresser la liste des cinq fonctions principales qu'un dictionnaire d'(auto-)apprentissage doit assumer : réception ; production; (auto-)apprentissage ; dimension lexiculturelle et médiation entre L1 et L2, L3, etc.. En passant en revue les dictionnaires de langue française récents, les auteurs révèlent les carences et les lacunes des dictionnaires de français langue maternelle Ils mentionnent également les influences internationales et les caractéristiques de dictionnaires pour apprenants de langue étrangère, élaborés dans d'autres pays. Sont présentées ensuite les innovations principales introduites par le DAFA ( Dictionnaire d'Apprentissage du Français des Affaires, version papier et électronique) et le DAFLES (Dictionnaire d’Apprentissage du Français Langue Etrangère ou Seconde), qui n'existe pour l'instant que sous forme électronique, consultable en ligne. Après se trouvent analysées les conditions pour pratiquer la lexicographie pédagogique. Dans les conclusions les auteurs soulignent les relations entre la lexicographie en langue maternelle et en langue étrangère et l'importance d'initier les apprenants de langues à la manipulation de dictionnaires, à l'analyse des différents types de dictionnaires et à leur structure. Enfin les auteurs évoquent les défis que doit relever le lexicographe qui souhaite réaliser un dictionnaire d' (auto-) apprentissage.
\end{abstract}

Mots clefs: lexicographie pédagogique, dictionnaires de (auto-)apprentissage, française-langue étrangère.

\section{RESUMO}

Neste texto, inicialmente, são expostas as cinco funções principais de um dicionário para aprendizes de língua estrangeira, a saber: recepção; produção; (auto-) aprendizagem; dimensão lexicultural e mediação. Em seguida, a partir de exemplos de dicionários de língua francesa, são discutidas as inadequações dos dicionários elaborados para falantes de língua materna ao serem utilizados por um público de aprendizes de língua estrangeira. Após serem assinaladas características de dicionários para estudantes de língua estrangeira produzidos em diferentes países, são expostas as inovações introduzidas pelos dicionários DAFA (Dictionnaire d'Apprentissage du Français des Affaires) nas versões em papel e eletrônica e DAFLES (Dictionnaire d'Apprentissage du Français Langue Etrangère ou Seconde), em versão eletrônica e, posteriormente, os autores analisam os requisitos necessários para a prática da lexicografia pedagógica. As conclusões dizem respeito às relações entre a lexicografia em língua estrangeira e em língua materna e à importância das práticas de uso do dicionário, de reflexão sobre seus diferentes tipos e da realização de exercícios sobre a estrutura dos dicionários nas aulas de línguas. Para finalizar, salienta-se o desafio do lexicógrafo ao realizar dicionários de (auto-) aprendizagem.

Palavras-chave: lexicografia pedagógica, dicionários de (auto-)aprendizagem, francês - língua estrangeira. 


\title{
INTRODUCTION
}

Avec les dictionnaires Larousse, Robert, Flammarion et Hachette, pour ne citer que les produits phare, et surtout le TLF, le joyau de la lexicographie française, le français dispose d'excellents dictionnaires encyclopédiques et de langue, surtout pour un public de français langue maternelle (FLM). Ils s'inscrivent dans une longue et prestigieuse tradition lexicographique.

A première vue on pourrait donc croire que tout a déjà été fait. Or, beaucoup reste à faire, comme le dit F.J. Hausmann $(1999,136)$ :

On vient de boucler la grande entreprise du TLF. 25000 pages pour décrire le vocabulaire français... Il n'y a aucun dictionnaire au monde qui égale le TLF. Et pourtant pour le traitement des locutions, tout reste à faire. (...) Et pour les collocations, une grande partie du travail n'est pas fait .

L'objectif de notre contribution est triple. Nous aimerions d'abord relever les influences étrangères dont la lexicographie du FLE, telle que nous la pratiquons en Flandre, est tributaire. Ensuite nous voudrions proposer quelques innovations dictionnairiques, que nous avons été amenés à introduire pour mieux satisfaire les besoins de nos apprenants. Enfin nous nous proposons de montrer que des synergies s'imposent entre la lexicographie du FLM et du FLE.

\section{LA LEXICOGRAPHIE PEDAGOGIQUE AU SERVICE DES APPRENANTS DE FLE: BESOINS ET DIFFICULTES}

\begin{abstract}
La lexicographie pédagogique (pour allophones) est une sous-branche de la lexicographie qui s'intéresse à l'étude et à la conception de dictionnaires dont l'objectif est de répondre aux besoins des apprenants d'une langue qui n'est pas leur langue maternelle. Cette branche particulière est assez complexe puisqu'elle se situe au carrefour de plusieurs disciplines comme la lexicologie, la psychologie, la didactique. Il faut combiner des champs différents et tenir compte des recherches effectuées dans chacun. Le lexicographe qui souhaite réaliser un dictionnaire d'apprentissage doit être un enseignant de langue étrangère, cumuler la fonction d'enseignant et de lexicographe afin de savoir quel vocabulaire doit être présenté à l'apprenant, comment le sélectionner, l'organiser, le présenter sur le plan linguistique.
\end{abstract}

Il importe donc que le lexicographe connaisse de façon aussi précise que possible les besoins et les difficultés des apprenants.

\section{Identification des besoins communicatifs des apprenants de FLE}

Les besoins d'apprentissage, de communication et d'autonomisation de ces apprenants sont spécifiques, comme l'a clairement démontré Tono (2001, 58, notre traduction): 
Trab.Ling.Aplic., Campinas, 44(2) - Jul./Dez. 2005

D'abord la distinction entre locuteurs natifs et allophones d'une langue-cible déterminée est cruciale. Les recherches ont montré que les besoins spécifiques des locuteurs natifs, comme utilisateurs de dictionnaires diffèrent considérablement de ceux de locuteurs allophones, qui sont avant tout des apprenants.

Pour répondre à ces besoins les dictionnaires d'(auto-)apprentissage qui leur sont destinés, comme le Dictionnaire d'Apprentissage du Français des Affaires (DAFA) et le Dictionnaire d'Apprentissage du Français Langue Etrangère ou Seconde (DAFLES) ${ }^{1}$, se doivent d'être multifonctionnels, et ce à plus d'un titre. Ils doivent assumer plusieurs fonctions et faire office de dictionnaires des collocations, des synonymes, des antonymes, des difficultés lexicologiques et grammaticales, d'un thésaurus.

En outre, ils devraient réunir les qualités essentielles relevées par Bogaards (1996 et 1998), c'est-à-dire l'intelligibilité, l'utilité et la convivialité. Nous y ajouterons également l'accessibilité, la flexibilité et l'interactivité pour ce qui est des versions électroniques de ces dictionnaires.

Les cinq fonctions principales qu'un dictionnaire d'(auto-)apprentissage doit assumer sont les suivantes:

1. réception, décodage, dépannage, pour reprendre la terminologie de Galisson (1987) qui distingue la lexicographie de dépannage de la lexicographie d'apprentissage. Le décodage constitue la fonction la plus traditionnelle, que les dictionnaires de FLM assument déjà. C'est beaucoup moins le cas pour les quatre fonctions suivantes.

2. production, encodage, mise en discours, ce qui implique qu'il faut développer la compétence combinatoire (lexicale), offrir une microstructure très riche, présenter le vocabulaire dans son fonctionnement discursif, offrir de nombreux contextes éclairants, prototypiques.

\section{3. (auto-)apprentissage}

Les dictionnaires constituent un outil extrêmement important dans le processus d'apprentissage autonome et le développement de cette autonomie s'avère une nécessité, si le professeur n'est plus présent. (Campo Cubillo 2002, 225, notre traduction)

Le dictionnaire (auto-)apprentissage doit pouvoir faire office de conseiller personnel et en quelque sorte remplacer le locuteur natif absent.

\section{4. dimension lexiculturelle}

Tout le monde se souvient de la petite phrase qui fut à l'origine du Dictionnaire des noms de marques courants de Galisson et André (1998) : «Deux «Vache qui rit », un coup de « Badoit » et ça repart, parfaitement compréhensible par un locuteur français,

${ }^{1}$ Les versions électroniques de ces dictionnaires sont accessibles aux adresses suivantes : DAFA (www.projetdafa.net); DAFLES (www.kuleuven.ac.be/dafles). 
mais d'une déroutante opacité pour de nombreux professeurs de français étrangers qui maîtrisent pourtant parfaitement bien la langue française ! Comme Schneider $(1998,266)$ le dit très bien:

Il s'agit de fournir à l'apprenant d'une langue étrangère ou seconde tous les outils dont il a besoin pour passer d'un discours, mais aussi d'un système culturel et conceptuel qui lui sont familiers au discours et au système culturel de la langue-cible qu'il connaît peu ou beaucoup moins.

\section{5. médiation}

Les dictionnaires d'(auto-)apprentissage assument également la fonction de médiation entre la langue-source et la langue-cible et devraient par conséquent être semi-multilingues, avec toute la nomenclature française traduite en plusieurs langues (l'anglais, l'allemand, l'espagnol, l'italien, etc.) pour avoir accès à la langue-cible par le biais de la langue maternelle, grâce aux index inversés. On pourrait pousser le multilinguisme jusqu' à fournir également toutes les traductions des unités polylexicales.

Plusieurs raisons militent en faveur du recours à la langue maternelle comme voie d'accès à la langue-cible :

On incite souvent les étudiants universitaires à utiliser des dictionnaires monolingues de la languecible, afin de les familiariser avec cette langue-cible, mais en réalité ils persistent à utiliser des dictionnaires bilingues parce que l'équivalent dans leur langue maternelle les rassure quant à l'information obtenue. (Campo Cubillo 2002, 222, notre traduction)

Koren (1997, 5, notre traduction) abonde dans le même sens :

L'usage d'un dictionnaire semi-bilingue semble constituer un compromis entre deux visions, celle qui privilégie le dictionnaire monolingue (le point de vue du professeur) et celle qui favorise le dictionnaire bilingue (le point de vue de l'apprenant).

En fait, la précieuse valeur ajoutée du dictionnaire semi-multilingue est qu'il combine à la fois les avantages du monolingue et du bilingue.

\section{Identification des difficultés des apprenants de FLE}

La fréquentation quotidienne du discours de nos apprenants de FLE néerlandophones, de leur interlangue, ainsi que l'analyse récente de leurs erreurs les plus fréquentes révèle qu' ils ont des problèmes aussi bien avec les collatéraux (p. ex. *importeur, *embauchement, *modieux) qu'avec les collocations (p. ex. un prix *haut, *demander une question), qui ne sont pas transposables d'une langue à l'autre. Parmi les corrélés, ce sont surtout les synonymes qui sont difficiles à maîtriser (p. ex. argent, fric, monnaie, fonds, liquidités, ressources, trésorerie). Remarquons que même dans les excellents dictionnaires d'apprentissage qui existent pour l'anglais, le traitement des collocations et des synonymes demeure une carence frappante (Bogaards 1996: 302-304 et 308-309). 
Parmi les autres difficultés majeures, signalons la maîtrise des informations pragmatiques. Dans quelle situation de communication emploie-t-on licencier, dégraisser les effectifs, virer, balancer, limoger ou, dans un autre contexte, crécher, demeurer, habiter ? Comment savoir que réajustement signifie tantôt une dévaluation dans réajustement monétaire, tantôt presque toujours une augmentation dans réajustement des salaires, alors que le dictionnaire de FLM se contente de la mention «action de réajuster» pour expliquer le nom déverbal de réajuster?

N'insistons pas sur la difficulté pour l'apprenant de FLE d'avoir accès à certaines informations, comme les unités polylexicales. Il faut déjà une certaine expertise et surtout beaucoup de courage pour retrouver dans un dictionnaire papier une expression courante, comme avoir le cceur sur la main, alors que l'on ne dispose pas de repères sémantiques, qui structurent clairement les articles COEUR et MAIN, qui peuvent occuper plusieurs colonnes dans un dictionnaire.

\section{INADEQUATION DES DICTIONNAIRES DE FLM QUI S'ADRESSENT A UN PUBLIC DE FLE}

C'est l'inadéquation des dictionnaires de FLM pour un public de FLE, le déficit dictionnairique qui conduit souvent au sentiment de déception, de dépit dictionnairique chez cette catégorie d'utilisateurs.

Dans l'aperçu ci-dessous, nous visualisons les principales différences entre les dictionnaires de FLM et de FLE.

\section{Différences dictionnaires FLM et FLE}

\begin{tabular}{|c|c|}
\hline $\begin{array}{l}\text { FLM } \\
\text { a.principes-clefs }\end{array}$ & FLE \\
\hline $\begin{array}{l}\text { Pour le locuteur natif tout va de soi, tout est } \\
\text { évident, prévisible }\end{array}$ & $\begin{array}{l}\text { Pour l'apprenant d'une langue étrangère rien ne } \\
\text { va de soi, rien n'est prévisible }\end{array}$ \\
\hline $\begin{array}{l}\text { intuition du locuteur natif } \\
\text { bonne capacité inférertielle, prévisionnelle } \\
\text { exposition, pratique permanentes }\end{array}$ & $\begin{array}{l}\text { intuition faibleou absente } \\
\text { faible capacité inférentielle, prévisionnelle } \\
\text { exposition, pratique limitées, intermittentes }\end{array}$ \\
\hline $\begin{array}{l}\text { b. fonctions } \\
\text { dépannage } \\
\text { bonne capacité inférentielle, prévisionnelle } \\
\text { particularités de la langue }\end{array}$ & $\begin{array}{l}\text { (auto-)apprentissage } \\
\text { décodage = encodage } \\
\text { caractéristiques essentielles, régularités, de la langue }\end{array}$ \\
\hline
\end{tabular}




\begin{tabular}{l|l}
\hline $\begin{array}{l}\text { FLM } \\
\text { c. macrostructure } \\
\text { plus d'entrées } \\
\text { classement sémasiologique, alphabétique } \\
\text { macrostructure }>\text { microstructure }\end{array}$ & FLE \\
$\begin{array}{l}\text { d. } \text { microstructure } \\
\text { plus de sens } \\
\text { définitions encyclopédiques, ouencyclopédisantes d'entrées } \\
\text { évocation, accumulation des synonymes (approche } \\
\text { cumulative, quantitative) } \\
\text { expressions grand nombre } \\
\text { collocations: peu nombreuses, aléatoires } \\
\text { peu de notes d'usage }\end{array}$ & $\begin{array}{l}\text { microstructure > macrostructure } \\
\text { définitions descriptives } \\
\text { différenciation des synonymes (approche distinctive, } \\
\text { contextuelle, contrastive) }\end{array}$ \\
\hline
\end{tabular}

Les dictionnaires de FLM, et de langue maternelle en général d'ailleurs, ne prennent pas, ne peuvent pas suffisamment bien prendre en compte les besoins, les difficultés, les habitudes de consultation du public FLE, puisque ces dictionnaires s'adressent à un public de locuteurs natifs. Hausmann $(1999,135)$ fait bien ressortir cette différence de perspective lorsqu'il écrit:

Le lexique n'est pas compositionnel, il est idiomatique, c.-à-d. labyrinthique au possible. Le locuteur natif au bout d'une quinzaine d'années passées dans ce labyrinthe a fini par se fabriquer le fil d'Ariane qui aide à s'y reconnaître, à s'y mouvoir sans difficultés, à s'y sentir à l'aise, au point de ne plus avoir conscience d'être dans un labyrinthe. Il suffit d'être étranger à ce labyrinthe, d'avoir grandi dans un autre labyrinthe, tout aussi compliqué et tout aussi immense, et de vouloir avec son propre fil d'Ariane remonter le labyrinthe étranger, pour s'apercevoir que tout est différent et qu'il faut repenser l'apprentissage à zéro.

\section{ADAPTATION DE L'OUTIL AU PUBLIC}

Pour que « l'objet se rapproche du sujet », il ne suffit donc pas d'adapter un dictionnaire de FLM au public FLE en procédant à une réduction quantitative de la macrostructure, en simplifiant quelque peu les définitions ou les phrases-exemples ou de mettre sur la couverture qu'il s'adresse également à un public de FLE. En fait, il faut effectivement « repenser l'apprentissage à zéro », concevoir de nouveaux types de dictionnaires, s'inspirer de toutes sortes de sources, que ce soit dans le domaine de la lexicographie monolingue ou plurilingue, française ou internationale, faire la synthèse de nombreuses disciplines, imaginer des solutions originales, recourir aux outils informatiques et aux technologies de l'information et de la communication pour l'enseignement (TICE), se baser sur son expérience didactique en tant que professeurs/lexicographes. Bref, un véritable changement de paradigme s'impose. 
Trab.Ling.Aplic., Campinas, 44(2) - Jul./Dez. 2005

\section{Influences internationales}

Reconnaissons d'abord notre dette envers un certain nombre d'influences étrangères avant d'évoquer quelques innovations dictionnairiques que nous avons introduites en élaborant le DAFA et le DAFLES. En dehors de notre noyau de lexicographes/dictionnaristes néerlandophones, nous avons collaboré dans le passé et collaborons toujours avec un certain nombre de collègues étrangers et français en particulier ${ }^{2}$. C'est surtout par le biais des contacts personnels, des publications, des colloques et congrès, comme les Journées du dictionnaire et les congrès EURALEX, par exemple, que notre équipe reste en contact avec les autres membres de la communauté scientifique internationale.

\section{La lexicographie française}

Il va de soi que tout professeur de français, que ce soit de FLM ou de FLE, en Flandre ou ailleurs, se trouve influencé par la formation qu'il a reçue et par les dictionnaires qu'il a été amené à consulter.

C'est ainsi que Goosse nous a révélé les avantages de l'approche onomasiologique.

Dans l'ouvrage Les dictionnaires de langue française. Dictionnaires d'apprentissage. Dictionnaires spécialisés de la langue. Dictionnaires de spécialité, Pruvost (2001) dresse l'inventaire des nombreuses réalisations et innovations de la lexicographie française, dont nous sommes tous quelque peu tributaires.

A vrai dire, c'est la lecture d'un article de Galisson (1987), intitulé 'De la lexicographie de dépannage à la lexicographie d'apprentissage', qui a fait office d'amorce dictionnairique. L'auteur y plaidait pour une réhabilitation du dictionnaire monolingue et pour que les dictionnaires assument une fonction nouvelle d'apprentissage à côté de leur traditionnelle fonction de dépannage. C'est à lui que nous avons emprunté la notion même de dictionnaire d'apprentissage, connue depuis bien plus longtemps dans le domaine anglo-saxon.

\section{L'influence du Dictionnaire explicatif et combinatoire du français contemporain (DEC) de Mel'èuk}

Le DAFA et le DAFLES doivent aussi beaucoup aux travaux de Mel'èuk dont nous nous sommes inspirés pour:

- les définitions actancielles, c'est-à-dire des définitions qui indiquent explicitement les différents arguments du prédicat ou, en d'autres termes, les participants à l'action ou à l'état,

- le principe de la décomposition sémantique,

- la standardisation de la structure de la définition et de ses composants,

\footnotetext{
${ }^{2}$ Pour les variantes géographiques du DAFA, par exemple, nous avons fait appel à des collègues d'autres pays de la francophonie: des collègues français, québecoises, suisse. Pour les traductions nous avons fait appel à des collègues anglais, allemand, espagnol, italien. Pour ce qui est du DAFA et du DAFLES, des collègues et des étudiants français des universités de Grenoble, de Lille, de Nanterre et d'Ottawa y collaborent activement.
} 
- l'organisation sémantique des collocations grâce aux fonctions lexicales. (voir e.a. Mel'èuk et al. 1995).

Il va sans dire qu'il a fallu procéder à une didactisation poussée des principes de Mel'èuk, pour qu'ils soient applicables dans le domaine de la lexicographie pédagogique et compréhensibles par un public de FLE. Leur utilisation s'est cependant avérée d'une richesse insoupçonnée.

\section{Les dictionnaires pour apprenants d'anglais}

Ce sont incontestablement les dictionnaires pour apprenants d'anglais, les fameux learner's dictionaries ${ }^{3}$, qui demeurent les prototypes du genre et dont nous sommes inspirés le plus. Dans son étude, Cowie (1999) en a retracé l'histoire et a montré le rôle-clef qu'a joué le père-fondateur de la lexicographie pédagogique Hornby.

Le DAFA et le DAFLES se situent dans la lignée de ces learners'dictionaries anglais et ils leur doivent un certain nombre de pistes d'optimisation:

- l'utilisation d'un corpus pour fournir une fréquence objective et pas seulement intuitive, et pour établir la nomenclature,

- l'organisation de la nomenclature, qui s'est inspirée du Longman Language Activator (LLA) : classement mixte, c'est-à-dire classement sémasiologique, alphabétique de la macrostructure et classement onomasiologique de la microstructure,

- le principe du vocabulaire définitoire contrôlé, bien que Matoré l'appliquât déjà en 1970 dans son Dictionnaire du vocabulaire essentiel, ${ }^{4}$

- les repères sémantiques qui indiquent l'architecture d'un article et en facilitent l'accès,

- les définitions phrastiques du style Cobuild (p. ex. pour expliquer l'adjectif malade : Si vous êtes malade, vous ne vous sentez pas bien, vous ne pouvez pas aller à l'école,...) au lieu de Se dit d'un être vivant dont l'organisme souffre de troubles, qui a subi une altération de sa santé, comme dans le Lexis.

\section{Les innovations principales introduites par le DAFA et le DAFLES}

Passons en revue les principales innovations dictionnairiques introduites par le DAFA et le DAFLES:

${ }^{3}$ Le Cambridge International Dictionary of Contemporary English (CIDE), le Collins Cobuild English Dictionary (COBUILD), l'Oxford Advanced Learner's Dictionary of current English (OALDE), le Longman Dictionary of Contemporary English (LDOCE), Macmillan English Dictionary for advanced learners (MACMILLAN) et le Longman Language Activator, (LLA)

${ }^{4}$ « Ce dictionnaire de conception entièrement nouvelle comprend les 5000 mots fondamentaux de la langue française, définis à l'aide de ce même vocabulaire de 5000 mots », comme l'atteste le texte publicitaire paru dans la revue Langue française, $\mathrm{n}^{\circ}$ 8, décembre 1970. 
- la multifonctionnalité : prise en compte des fonctions lexiculturelle, de décodage, d'encodage, d'apprentissage et de médiation,

- le regroupement de toutes les informations lexicographiquement pertinentes, dont l'apprenant de FLE a besoin pour communiquer dans la langue étrangère : informations morpho-syntaxiques, syntagmatiques, paradigmatiques et pragmatiques,

- l'organisation systématique à la fois linguistique et conceptuelle,

- l'organisation onomasiologique de la microstructure qui permet un regroupement structuré des termes associés, si importante pour l'ancrage cognitif et pour la mise en discours,

- la différenciation systématique des synonymes et expressions synonymiques,

- les définitions longues et courtes qui permettent de doser l'information,

- la description systématique de la combinatoire des mots,

- l'organisation des collocations selon des critères à la fois morphosyntaxiques, sémantiques et pragmatiques,

- l'enregistrement des variantes géographiques avec l'indication des limites sémantiques et des contraintes d'usage, ainsi que l'attestation des principaux emplois métaphoriques, qui relèvent de la fonction lexiculturelle du dictionnaire,

- l'indication des aspects connotatifs, des contraintes morpho-syntaxiques,

- la multiplication des voies d'accès, entre autres par la langue maternelle (l'allemand, l'anglais, l'espagnol, l'italien, etc.) et la recherche par domaines dans les versions électroniques,

- la possibilité d'avoir accès aux combinaisons de mots par chacune de leurs composants,

- la didactisation poussée qui se manifeste par les nombreuses remarques et notes d'usage et par l'ampleur de certains articles, comme par exemple l'article FLUCTUATION dans le DAFA, qui fournit aux apprenants des critères de sélection et de restriction introuvables dans les autres dictionnaires.

- les schémas actanciels qui visualisent et explicitent de façon systématique tous les actants et circonstants des verbes. Ils apportent une valeur ajoutée considérable par rapport aux traditionnels tableaux de dérivation qui ne retiennent que les dérivés morphosémantiquement pertinents.

Cette didactisation fait du dictionnaire un instrument d'autonomisation et de responsabilisation de l'apprenant.

Par le regroupement de tant d'informations diverses, le DAFA et le DAFLES peuvent facilement faire office de générateur d'exercices (Verlinde, Selva et Binon, 2002), de matrice pour un auto-dictionnaire personnalisé et constituer le noyau d'un environnement d'apprentissage puissant. 
Remarquons que les multiples fonctionnalités de la version électronique de nos dictionnaires d'(auto-)apprentissage offrent plusieurs avantages par rapport à la version papier, du moins dans la mesure où il s'agit de dictionnaires électroniques d'une nouvelle génération, et non de la transposition sur support électronique de dictionnaires papier :

- une consultation différenciée selon les besoins de réception ou de production,

- un confort d'usage par un accès beaucoup plus rapide et bien plus efficace aux informations, surtout aux unités polylexicales,

- une plus grande flexibilité d'usage qui permet de suivre un parcours individualisé,

- un plus grand contrôle des informations affichées par le biais de l'interactivité,

- une organisation réticulaire des (para)synonymes ou des expressions synonymiques et des dérivés-actants,

- la possibilité de suivre le parcours individuel de chaque utilisateur grâce à un programme de traces.

Avec Rundell (1998, 325-337, notre traduction), il y a lieu de remarquer que

Tout ceci reflète un changement de paradigme bienvenu, le passage d'un modèle de dictionnaire pour locuteurs natifs inadéquat vers une lexicographie plus utilitaire qui fait prévaloir avant tout les besoins des utilisateurs.

\section{CONDITIONS POUR PRATIQUER LA LEXICOGRAPHIE PEDAGOGIQUE DU FLE}

Pour pratiquer la lexicographie du FLE, telle que nous venons de la décrire et faire des dictionnaires multifonctionnels, plusieurs conditions doivent être remplies. Il faut

- connaître les besoins communicatifs, les difficultés des apprenants, donc disposer d'une solide expérience didactique,

- connaître leurs habitudes de consultation. Pour ce faire, il est nécessaire de procéder à des enquêtes ou, mieux encore, d'observer les apprenants en les soumettant à des tests (Selva et Verlinde, 2002),

- avoir une idée des processus d'apprentissage du vocabulaire (importance d'une organisation à la fois conceptuelle et linguistique, de l'ancrage cognitif), des processus cognitifs mis en oeuvre lors de la consultation d'un dictionnaire,

- avoir une idée de la façon dont le vocabulaire est enseigné,

- disposer d'un corpus suffisamment représentatif,

- posséder une solide expertise lexicographique, 
- procéder à une didactisation de plus en plus poussée: «l'objet » a tout intérêt à « se rapprocher du sujet », comme le dirait Galisson, à y coller même.

Bref, il s'agit de pratiquer la pluridisciplinarité et la transdidacticité, c'est-à-dire le transfert de savoir-faire didactique d'un domaine à un autre, d'une langue à une autre.

\section{CONCLUSIONS}

\section{Aucun dictionnaire n'est parfait}

Non, aucun dictionnaire n'est parfait et il ne nous incombe pas de dresser un palmarès des meilleurs dictionnaires. Les learner's dictionaries anglais continuent à juste titre d'être considérés comme les modèles des dictionnaires pour apprenants. Le LDOCE, par exemple, offre l'éventail le plus riche de marques d'usage et innove en proposant des graphiques qui visualisent le taux de fréquence de certaines unités lexicales, tout comme le SALAMANCA, le champion des marques diatopiques (19 !). Si le LANGENSCHEIDT excelle dans le traitement des antonymes, bien contextualisés, le DFU de son côté se distingue par la judicieuse organisation onomasiologique de sa microstructure, le DAFA et le DAFLES par la richesse des informations collocationnelles, par la visualisation et par l'explicitation des actants, le DAFA électronique et le DAFLES par la multiplicité des voies d'accès et par la facilité d'accès aux unités polylexicales. Le DAFLES innove en outre par ses schémas actanciels.

\section{Lexicographie FLE et FLM : des synergies s'imposent}

Dans la mesure où le dictionnaire de FLM s'adresse à de jeunes apprenants francophones, qui ne maîtrisent pas encore parfaitement leur langue maternelle, il aurait peut-être intérêt à mieux assumer la fonction d'apprentissage, par une organisation à la fois conceptuelle et linguistique plus poussée, ainsi que la fonction d'encodage, par une microstructure plus riche. Bref, la multifonctionnalité qui vaut pour le FLE nous paraît tout aussi valable pour le FLM. Ce repositionnement entraînerait la mention obligatoire et plus systématique d'un certain nombre d'informations morphologiques (les principaux dérivés morphosémantiquement pertinents, les noms déverbaux), sémantiques (des définitions plus simples, moins circulaires, actancielles) morphosyntaxiques (valence des verbes, mais aussi des noms et des adjectifs), syntagmatiques (attestation et organisation sémantique des principales collocations) et paradigmatiques (différenciation systématique des (para)synonymes, indication des règles d'usage). Une approche contextuelle, distinctive devrait peut-être se substituer à l'approche cumulative, encore souvent en vigueur actuellement dans les dictionnaires de FLM. Ceux-ci se contentent d'habitude de donner les synonymes en vrac, sans les relier à chacune des acceptions d'un mot ou d'une unité lexicale et sans préciser les critères de sélection ou de restriction. Ceci ne pose sans doute 
pas de problèmes pour le locuteur natif averti, pour qui il suffit de rappeler l'existence de certains synonymes, mais peut constituer un handicap pour une jeune élève francophone.

Les notes d'usage auraient intérêt à être développées davantage, à ne pas se limiter à attirer l'attention sur des problèmes d'orthographe, mais à inclure un certain nombre d'informations pragmatiques, dont le taux de fréquence objective. Nous avons en effet l'impression qu'en faisant l'impasse sur toute une série de données, comme par exemple les collocations, un nombre suffisant de phrases-exemples, les constructions syntaxiques, la différenciation systématique des (para)synonymes et l'indication de la fréquence, les dictionnaires de FLM font trop confiance à l'intuition du locuteur natif. De plus en plus d'apprenants sortent de milieux défavorisés et connaissent de nombreux problèmes lors de leur scolarisation. Dans certaines écoles de FLM se trouvent d'ailleurs pas mal d'apprenants de français langue seconde. Il nous semble donc que la lexicographie du FLM aurait tout intérêt à s'engager dans la voie de la didactisation et de la multifonctionnalité, deux pistes d'optimisation potentielles intéressantes.

S'il paraît tout indiqué que les dictionnaires de FLM adoptent certaines innovations dictionnairiques du FLE, il va de soi que les dictionnaires de FLE, de leur côté, auraient intérêt à enrichir leur nomenclature, à intégrer davantage le français parlé, familier, souvent sous-représenté, faute de corpus du français oral, facilement accessibles.

\section{IL faut initier les apprenants à la manipulation du dictionnaire}

Selon Sobrinho (1998) et Tono (2001, 3), le dictionnaire peut seulement constituer une aide considérable, si l'enseignement réserve une partie de son temps à l'initiation à l'emploi du dictionnaire. Il faut informer les étudiants des qualités et des défauts des différents types de dictionnaires, leur en faire découvrir toute la richesse, prévoir des exercices concernant leur structure et surtout stimuler l'emploi des différents types de dictionnaires.

Il est évident, comme l'écrit Vigner (1983, 190), en faisant allusion aux innovations dans le domaine de la lexicographie pédagogique, que

\footnotetext{
toutes ces propositions n'ont qu'un faible caractère de cohérence en regard d'une théorie linguistique. Elles trouvent leur seule justification dans le souci de définir un ouvrage à visée pratique au plan didactique. Le jeu d'usage qui est actuellement fait des dictionnaires monolingues dans l'apprentissage de la langue (...) ou du moins le faible rendement (...) plaide rigoureusement pour une remise en question d'outils qui doivent trouver leur justification non pas par référence à des théories linguistiques (toujours inachevées et souvent contradictoires) qu'ils seraient censés refléter, mais par la qualité du service qu'ils peuvent rendre aux apprenants.
}

Nous sommes convaincus que la collaboration entre le professeur/lexicographe de FLE, de FLM et d'autres langues peut donner lieu à une fertilisation croisée tout à fait productive. Signalons d'ailleurs que les principes décrits ci-dessus nous paraissent valables, non seulement pour le FLE, mais également pour d'autres langues ayant le statut de langue étrangère ou seconde. 
Trab.Ling.Aplic., Campinas, 44(2) - Jul./Dez. 2005

Nous sommes également persuadés que pour être vraiment efficace et crédible il faut que le lexicographe se double d'un didacticien averti, qui s'intéresse à la façon dont s'enseigne et s'apprend le vocabulaire et dont l'expérience permet d'entrevoir les difficultés, les désirs et les besoins des lecteurs.

Pour pratiquer une lexicographie pédagogique au service des apprenants et réaliser des dictionnaires d'(auto-)apprentissage susceptibles de les aider vraiment à résoudre leurs problèmes de communication, il importe d'intégrer de façon critique les acquisitions les plus récentes de la lexicographie internationale. Qui plus est, il faut oser s'affranchir des modèles et des procédés existants, s'aventurer hors des sentiers dictionnairiques battus, innover, voire même oser jouer un rôle de pionnier.

L'avantage du professeur qui enseigne le FLE et qui travaille à la périphérie de la francophonie, ne serait-il pas de poser sur la langue, et sur le vocabulaire en particulier, un regard différent qui lui permet de mieux appréhender les problèmes des apprenants et peut-être de proposer des remèdes? Pour que «l'objet se rapprochât du sujet » un changement de paradigme s'imposait. Dorénavant ce changement ne relève plus de la lexicographiction!

\section{REFERENCES BIBLIOGRAPHIE}

\section{A. Dictionnaires papier}

PROCTER, P. (éd.) (1995). Cambridge International Dictionary of Contemporary English (CIDE). Cambridge: Cambridge University Press.

SINCLAIR, J. (éd.) (1995). Collins Cobuild English Dictionary (COBUILD). London: Harper Collins.

CORRÉARD, M.H. (1997). The Oxford-Hachette French Dictionary. Paris/Oxford: Hachette/Oxford University Press.

CUADRADO, J.G. et al. (1996). Diccionario Salamanca de la lengua española (SALAMANCA). Universidad de Salamanca.

BINON, J., VERLINDE, S., VAN DYCK, J. et BERTELS, A. (2000). Dictionnaire d'apprentissage du français des affaires (DAFA). Paris: Didier.

VERLINDE, S., BINON, J., BERTELS, A., VAN DYCK, J. et Selva, T. (en préparation) Dictionnaire d'apprentissage du français langue étrangère ou seconde (DAFLES). Louvain-la-Neuve, De Boeck.

MEL'ÈUK, I. et al. (1984, 1988, 1992 et 1999). Dictionnaire explicatif et combinatoire du français contemporain: Recherches lexico-sémantiques (DEC) I, II, III et IV. Montréal: Presses de l’Université de Montréal.

PICOCHE, J. et ROLLAND, J.-Cl. (2002). Dictionnaire usuel du français (DFU). Bruxelles: De Boeck-Duculot.

EZQUERRA, M.A. et al., (1995). Diccionario para la enseñanza de la lengue española. Universidad de Alcalá de Henares.

FOURMENT, M. (1998). Dizionario di apprendimento della lingua francese (DAF). Torino: Paravia. 
BINON, VERLINDE, SELVA - Influences internationales...

GALISSON, R. et ANDRÉ, J.C. (1998). Dictionnaire des noms de marques courantes. Essai de lexiculture ordinaire. Paris: CNRS (INaLF)/Didier Erudition.

ILGENFRITZ, P. et al. (1989). Langenscheidts Kontextwörterbuch Französisch-Deutsch. Ein neues Wörterbuch zum Schreiben, Lernen, Formulieren (LANGENSCHEIDT). Berlin: Langenscheidt.

JEHLE, G. et al. (1998). Langenscheidts Grosswörterbuch Deutsch als Fremdsprache. Berlin/München : Langenscheidt.

SUMMERS, D. (éd.) (1995). Longman Dictionary of Contemporary English (LDOCE). Harlow: Longman.

ADAM, J.H. (1998). Longman Dictionary of Business English (LDBE). Harlow: Longman.

SUMMERS, D. (éd.) (1993). Longman Language Activator. The World's First Production Dictionary (LLA). Harlow: Longman.

MATORÉ, G. (1970). Dictionnaire du vocabulaire essentiel. Paris: Larousse.

CROWTHER, J. (éd.) (1995). Oxford Advanced Learner's Dictionary of current English (OALD). Oxford: Oxford University Press.

TUCK, A. (1998). Oxford Dictionary of Business English for learners of English (ODBE). Oxford: Oxford University Press.

REY-DEBOVE, J. et al. (1999). Dictionnaire du français. Référence/apprentissage. Paris: Le Robert/CLE International.

RUNDELL, M. (éd.) (2002). Macmillan English Dictionary for advanced learners (MACMILLAN). London: Macmillan.

\section{B. Dictionnaires électroniques}

(1996). Hachette-Oxford sur CD-ROM (HOCD). Oxford-Paris: Oxford University Press - Hachette.

REY, A. (1996). Le Petit Robert sur CD-ROM (PRCD). Paris: Dictionnaires Le Robert.

\section{Articles et études}

ABEL, A. et WEBER, V. (2000). ELDIT - A prototype of an innovative dictionary. In Heid, U., et al. (éds) Proceedings EURALEX, The Ninth EURALEX International Congress. Stuttgart: Universität Stuttgart. 807-818.

ATKINS, B.T.S. et VARANTOLA, K. (1997). 'Monitoring dictionary use'. International Journal of Lexicography 10.1: 1-45.

BINON, J., DANCETTE, J. et VERLINDE, S. (1998). 'Comment améliorer le traitement des synonymes dans un dictionnaire de langue'. In: Fontenelle, Th. et al. (éds) Actes Euralex'98 Proceedings. (Communications soumises à Euralex'98 (Huitième congrès international de lexicographie) à Liège, Belgique). Vol. I: $77-$ 86. 
BINON, J., SELVA, T. et VERLINDE, S. (à paraître) 'Tendances et innovations récentes en lexicographie pédagogique. La contribution du DAFA et du DAFLES'. Actes du Symposium internacional de Lexicografia, organisé à Barcelone par la Universitat Atutonoma de Barcelone, l'Universitat Rovira $i$ Virgili de Lleida et l'Institut d'Estudis Catalans.

BINON, J. et VERLINDE, S. (1998). 'Le Dictionnaire d'apprentissage du français des affaires: un nouveau type de dictionnaire d'apprentissage multilingue axé sur la production'. Le langage et l'homme 33.4 : 437-449.

BINON, J. et VERLINDE, S. (1999). 'La contribution de la lexicographie pédagogique à l'apprentissage et à l'enseignement d'une langue étrangère ou seconde'. Etudes de linguistique appliquée 116 : 453-468.

BINON, J., VERLINDE, S. et SELVA, T. (2001). 'Lexicographie pédagogique et enseignement/apprentissage du vocabulaire en français langue étrangère ou seconde (FLES). Un mariage parfait'. Cahiers de lexicologie $78.1: 1-23$.

BOGAARDS, P. (1991). 'Dictionnaires pédagogiques et apprentissage du vocabulaire'. Cahiers de lexicologie 59.2: 93-107.

BOGAARDS, P. (1996). 'Dictionaries for learners of English'. International Journal of Lexicography 9.4 : 277-320.

(1998). 'Des dictionnaires au service de l'apprentissage du français langue étrangère'. Cahiers de lexicologie 72.1 : 127-167.

. (1999). 'Access structures of learners' dictionaries'. In Herbst, T. et Popp, K. (éds) The Perfect Learners' Dictionary (?). Tübingen : Max Niemeyer Verlag. 113-131.

(2002). 'DAFA, an Innovative Learner's Dictionary of Business French'. International Journal of Lexicography $15.1: 105-117$.

CAMPO CUBILLO, M. C. (2002). 'Dictionary use and dictionary needs of ESP students: an experimental approach'. Internationaml Journal of Lexicography 15.3 : 206-229.

COWIE, A.P. (1999). English Dictionaries for Foreign Learners. A History. Oxford : Clarendon Press.

FOLTÊTE, I. (1994). L'expression de la mesure dans la langue du marketing. Thèse de doctorat non publiée. Université de Paris VII.

FUERTES-OLIVERA, P.A. et VELASCO-SACRISTAN, M. (2001). 'A critical comparison of the Macrostructure and Microstructure of two Bilingual English-Spanish Dict!ionaries of Economics' International Journal of Lexicography $14.1: 31-56$.

GALISSON, R. (1987). 'De la lexicographie de dépannage à la lexicographie d'apprentissage' Cahiers de lexicologie 51.2, 95-117.

(1999a). 'La pragmatique lexiculturelle pour accéder autrement à une autre culture par un autre lexique'. Etudes de Linguistique Appliquée 116: 447-496.

.( 1999b). 'Regards disciplinaires croisés sur l'accès à la maîtrise des vocabulaires'. Etudes de Linguistique Appliquée 116 : 389-394.

GUÉVEL, Z. (1995). La lexicographie française des affaires. Représentation lexicale d'une langue de spécialité. Thèse de doctorat non publiée. Université de Paris XII.

HAUSMANN, F. J. (1984). 'Wortschatzlernen ist Kollokationslernen'. Praxis des NU 31: 395-406. 
HAUSMANN, F. J. (1999). Le dictionnaire de collocations - Critères de son organisation“. In Greiner, N., Krnelius, J. et Rovere, G. Texte und Kontexte in Sprachen und Kulturen, Festschrift für Jörn Albrect Trier: WVT Wissenschaftlicher Verlag.

HERBST, T. et Popp, K. (1999). The Perfect Learners' Dictionary ( ?). Tübingen : Max Niemeyer Verlag.

HEID, U. (1994). 'On ways words work together - topics in lexical combinatorics'. In Martin, W. et al. (éds) Euralex 1994, Proceedings. Amsterdam : Vrije Universiteit. 226-257.

HOWARTH, P. (2000). 'Benson, Benson and Ilson, The BBI Dictionary of English Word Combinations'. International Journal of Lexicography $13.1: 50-54$

ILSON, R., (1999). 'Nine Learner's dictionaries'. International Journal of Lexicography 12.3: 223-237.

KLOTZ, M. (1999). 'Word complementation in English learners' dictionaries - a quantitative study of CIDE, COBUILD2, LDOCE3 and OALD5'. In Herbst, T. et Popp, K. (éds) The Perfect Learners' Dictionary (?). Tübingen : Max Niemeyer Verlag. 33-44.

KOREN, S., (1997). 'Quality versus convenience; Comparison of Modern dictionaries from the Researcher's, Teacher's and Learner's Point of view'. TESL-EJ 2.3.

LAUFER, B. et MELAMED, L. (1994). 'Monolingual, bilingual and 'bilingualised' dictionaries : which are more effective, for what and for whom?'. In Martin, W. et al. (éds) Euralex 1994, Proceedings. Amsterdam : Vrije Universiteit. 565-576.

LEECH, G. et NESI, H. (1999). 'Moving towards perfection : the learner's (electronic) dictionary of the future' . In Herbst, T. et Popp, K. (éds) The Perfect Learners’ Dictionary ( ?). Tübingen : Max Niemeyer Verlag. 295-306.

MEL'ÈUK, I., CLAS, A. et POLGUÈRE, A. (1995). Introduction à la lexicologie explicative et combinatoire. Louvain-la-Neuve: Duculot.

MITTMANN, B. (1999). 'The treatment of collocations in OALD5, LDOCE3, COBUILD2 and CIDE'. In Herbst, T. et Popp, K. (éds) The Perfect Learners'Dictionary( ?). Tübingen: Max Niemeyer Verlag. 101112.

NIKLAS-SALMINEN, A., (1997). La lexicologie. Paris: Colin.

PRUVOST, J., (éd.) (2001). Les dictionnaires de langue française. Dictionnaires d'apprentissage. Dictionnaires spécialisés de la langue. Dictionnaires de spécialité. Paris : Honoré Champion.

RUNDELL, M. (1998). 'Recent trends in pedagogical lexicography'. International Journal of Lexicography $11.4: 315-342$.

SCHAEFFLER, F. (2002). Aspects linguistiques et didactiques d'un dictionnaire d'Apprentissage en ligne : le cas du DAFLES. Mémoire de maîtrise. Université de Nanterre.

SCHNEIDER, F. (1998). Studien zur kontektuellen Fachlexicographie. Das deutsch-französische Wörterbuch der Rechnungslegung. Tübingen : Max Niemeyer Verlag.

SELVA, T. (1999). Ressources et activités pédagogiques dans un environnement informatique d'aide à l'apprentissage lexical du français langue seconde. thèse de doctorat non publiée. Université de FrancheComté. 
Trab.Ling.Aplic., Campinas, 44(2) - Jul./Dez. 2005

SELVA, T. et VERLINDE, S. (2002). 'L'utilisation d'un dictionnaire électronique : une étude de cas'. In Braasch, A. et Pulsen, C. (éds). Proceedings of the tenth EURALEX International Congress. Copenhagen. Vol II: 773-785.

SELVA, T., VERLINDE, S. et BINON, J. (2002). 'Le DAFLES, un nouveau dictionnaire pour apprenants du français'. In Braasch, A. et Pulsen, C. (éds) Proceedings of the tenth EURALEX International Congress. Copenhagen. Vol I: 199-208.

SOBRINHO, J.C., (1998). O dicionario como instrumento auxiliar na leitura em lingua estrangeira, Mémoire de licence. Universidade Federal de Minas Gerais, Belo Horizonte.

SVARTVIK, J. (1999). 'Corpora and dictionaries'. In Herbst, T. et Popp, K. (éds) The Perfect Learners' Dictionary ( ?). Tübingen: Max Niemeyer Verlag. 283-295.

SWANEPOEL, P. (2000). 'Providing lexicographic support for SL vocabulary acquisition : What kind, under what conditions, for whom and why?' In Heid, U. et al. (éds) Proceedings EURALEX, The Ninth EURALEX International Congress. Stuttgart: Universität Stuttgart. 403-419.

TONO, Y. (2001). Research on Dictionary Use in the Context of Foreign Language Learning, Focus on Reading Comprehension. Tübingen: Max Niemeyer Verlag.

VAN CAMPENHOUDT, M. (2002). 'Pour une Approche Sémantique du Terme et de ses Equivalents'. International Journal of Lexicography 14.3: 181-211.

VERLINDE, S. (1995). 'La combinatoire du vocabulaire des fluctuations dans le discours économique'. Cahiers de lexicologie 66.1: 137-176.

VERLINDE, S. (2002). 'Le DAFA, un dictionnaire d'(auto-)apprentissage du français des affaires, générateur d'exercices et d'activités de communication'. Point Commun 17, 27-30.

VERLINDE, S., DANCETTE, J. et BINON, J. (1998). Redéfinir la définition. In: Fontenelle, Th. et al. (éds). Actes Euralex'98 Proceedings. (Communications soumises à Euralex'98 (Huitième congrès international de lexicographie) à Liège, Belgique). Vol. II : 375-386.

VERLINDE, S. et SELVA, T. (2001). 'Nomenclature de dictionnaire et analyse de corpus'. Cahiers de lexicologie, 79. 113-139.

VERLINDE, S., SELVA, T. et BINON, J. (2002). 'Les dictionnaires électroniques et apprentissage du vocabulaire' In Binon, J. et al. (éds) Tableaux vivants, Opstellen over taal-en-onderwijs, aangeboden aan Mark Debrock. Leuven : Universitaire Pers. 179-201.

. (à paraître) 'Les collocations dans les dictionnaires d'apprentissage: repérage, présentation et accès'

VIGNER, G. (1983). 'Pour un nouveau dictionnaire'. Etudes de Linguistique Appliquée 49 : 174-191.

Sites Internet:

www.kuleuven.be/grelep (Groupe de recherche en lexicographie pédagogique)

www.projetdafa.net (Dictionnaire d'apprentissage du français des affaires)

www.kuleuven.be/dafles (Dictionnaire d'apprentissage du français langue étrangère ou seconde) 